\title{
Synthesis of 2-Acyloxycyclohexylsulfonamides and Evaluation on Their Fungicidal Activity
}

\section{Xinghai Li ${ }^{1, \dagger}$, Zining Cui ${ }^{2, \dagger}$, Xiaoyuan Chen ${ }^{1}$, Decai Wu ${ }^{1}$, Zhiqiu Qi ${ }^{1}$ and Mingshan Ji ${ }^{1{ }^{1} *}$}

1 Department of Pesticide Science, Plant Protection College, Shenyang Agricultural University, Shenyang 110866, Liaoning, China; E-Mails: lixinghai30@aliyun.com (X.L.); desk101@163.com (X.C.); wudecai@163.com (D.W.); syqizhiqiu@sina.com (Z.Q.)

2 Guangdong Province Key Laboratory of Microbial Signals and Disease Control, Department of Plant Pathology, College of Natural Resources and Environment, South China Agricultural University, Guangzhou 510642, Guangdong, China; E-Mail: ziningcui@scau.edu.cn

$\dagger$ These authors contributed equally to this work.

* Author to whom correspondence should be addressed; E-Mail: jimingshan@163.com; Tel.: +86-24-8849-2673; Fax: +86-24-8848-7148.

Received: 8 October 2013; in revised form: 31 October 2013 / Accepted: 4 November 2013 / Published: 14 November 2013

\begin{abstract}
Eighteen $N$-substituted phenyl-2-acyloxycyclohexylsulfonamides (III) were designed and synthesized by the reaction of $N$-substituted phenyl-2-hydroxylcycloalkylsulfonamides $\left(\mathbf{I}, \mathrm{R}^{1}\right)$ with acyl chloride (II, $\mathbf{R}^{2}$ ) in dichloromethane under the catalysis of TMEDA and molecular sieve. High fungicidal active compound $N$ - $(2,4,5-$ trichlorophenyl)-2-(2-ethoxyacetoxy) cyclohexylsulfonamide (III-18) was screened out. Mycelia growth assay against the Botrytis cinerea exhibited that $\mathrm{EC}_{50}$ and $\mathrm{EC}_{80}$ of compound III-18 were 4.17 and $17.15 \mu \mathrm{g} \mathrm{mL}^{-1}$ respectively, which was better than the commercial fungicide procymidone $\left(\mathrm{EC}_{50}=4.46 \mu \mathrm{g} \mathrm{mL}^{-1}\right.$ and $\left.\mathrm{EC}_{80}=35.02 \mu \mathrm{g} \mathrm{mL}^{-1}\right)$. For in vivo activity against $B$. cinerea in living leaf of cucumber, the control effect of compound III-18 was better than the fungicide cyprodinil. In addition, this new compound had broader fungicidal spectra than chlorothalonil.
\end{abstract}

Keywords: 2-acyloxycycloalkylsulfonamides; Botrytis cinerea; anti-fungal spectra; structure-activity relationship 


\section{Introduction}

Arylsulfonamides possessed excellent bioactivity against various kinds of bacteria and fungi, which were applied as medicines [1-8] and agrichemicals [9-13]. Recently, some novel arylsulfonamides containing different scaffolds, such as benzenesulfonamide (Figure 1A) [14] and the derivatives having 1,2,3-triazol (Figure 1B) [15], pyran ring (Figure 1C) [16], 1,6-caprolactam (Figure 1D) [17] and thiazolidinone (Figure 1E) [18] were reported for their obvious and diverse fungicidal activity against Sclerotinia sclerotiorum (Lib.) de Bary, Puccinia recondite f. sp. tritici, Phomopsis asparagi, Cladosporium fulvum and Fusarium oxysporum.

Figure 1. Structures of compounds A-M.<smiles>COC(=O)c1ccc(/N=C/c2cccc([N+](=O)[O-])c2)cc1S(N)(=O)=O</smiles>

A<smiles>CC(C)(C)/C(Cn1nccn1)=N\NS(=O)(=O)c1ccc(F)cc1</smiles>

B<smiles>CC(=O)Nc1ccc(S(=O)(=O)NC2COC(CCC(=O)O)CC2O)cc1</smiles>

C<smiles>[R]NOS(=O)(=O)C1CCCCCCCCCC1=O</smiles>

$\mathrm{F}: \mathrm{R}=4-\mathrm{ClC}_{6} \mathrm{H}_{4}$;

$\mathrm{G}: \mathrm{R}=\mathrm{CONH}\left(2,5-\mathrm{Cl}_{2} \mathrm{C}_{6} \mathrm{H}_{3}\right)$;<smiles>[R]NOS(=O)(=O)C1CCCCC1=O</smiles>

$\mathrm{H}: \mathrm{R}=2-\mathrm{CF}_{3}-4-\mathrm{ClC}_{6} \mathrm{H}_{3}$;

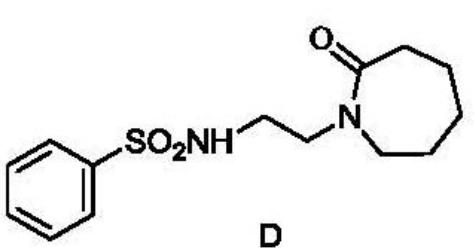

D<smiles>[R]c1ccc(S(=O)(=O)NC(=O)N2CCSC2=O)c([R])c1</smiles><smiles></smiles>

I: $\mathrm{R}=3-\mathrm{CF}_{3}-4-\mathrm{NO}_{2} \mathrm{C}_{6} \mathrm{H}_{3} ; \quad \mathrm{J}: \mathrm{R}=$ substituted phenyl and alkyl;<smiles>[R]NS(=O)(=O)C1CCc2ccccc2C1=O</smiles>

$\mathrm{K}: \mathrm{R}=3-\mathrm{CH}_{3} \mathrm{C}_{6} \mathrm{H}_{4}$;<smiles>[R]NOS(=O)(=O)C1CCCC1O</smiles>
$\mathrm{n}=1,2,3,4$

$\mathrm{L}: \mathrm{R}=2-\mathrm{CF}_{3}-4-\mathrm{ClC}_{6} \mathrm{H}_{3}$;

$\mathrm{M}: \mathrm{R}=2,4,5-\mathrm{Cl}_{3} \mathrm{C}_{6} \mathrm{H}_{2}$;

To explore new potential fungicides, some novel cycloalkylsulfonamides (Figure 1F-M) were designed and synthesized in our previous works, which had very good fungicidal activity, especially against the B. cinerea. 2-Oxocyclododecylsulfonamides (Figure 1F) [19] and (Figure 1G) [20] showed excellent in vitro activity against $B$. cinerea and Gibberella zeae (Schw.) Petch. When the ring was contracted to six-membered ring, the compounds (Figure 1H) [21], (Figure 1I) [22] with trifluoromethyl group and compound (Figure 1J) [23] also showed strong activities against B. cinerea and Sclerotinia sclerotiorum. The derivative of tetralonesulfonamide (Figure 1K) [24] exhibited good activity against $B$. cinerea and Rhizoctonia solani. When the carbonyl group was changed into hydroxyl group (Figure 1L) [25,26] and (Figure 1M) [27], the activity against B. cinerea was improved, not only in vitro but also in vivo. 
The ester group is the common bioactive moiety and 2-hydroxycycloalkylsulfonamides possess promising fungicidal activity. In continuation of our research on the synthesis of biological cycloalkylsulfonamide compounds, esterification was applied to the $N$-substituted phenyl-2hydroxycycloalkylsulfonamides (I), which was as the lead compounds to get the title compounds III (Scheme 1). Their fungicidal activity against $B$. cinerea and other 10 kinds of pathogenic fungi were evaluated to obtain the optimized compounds with significantly improved fungicidal activity and broad antifungal spectra.

Scheme 1. Synthetic route of compounds III.

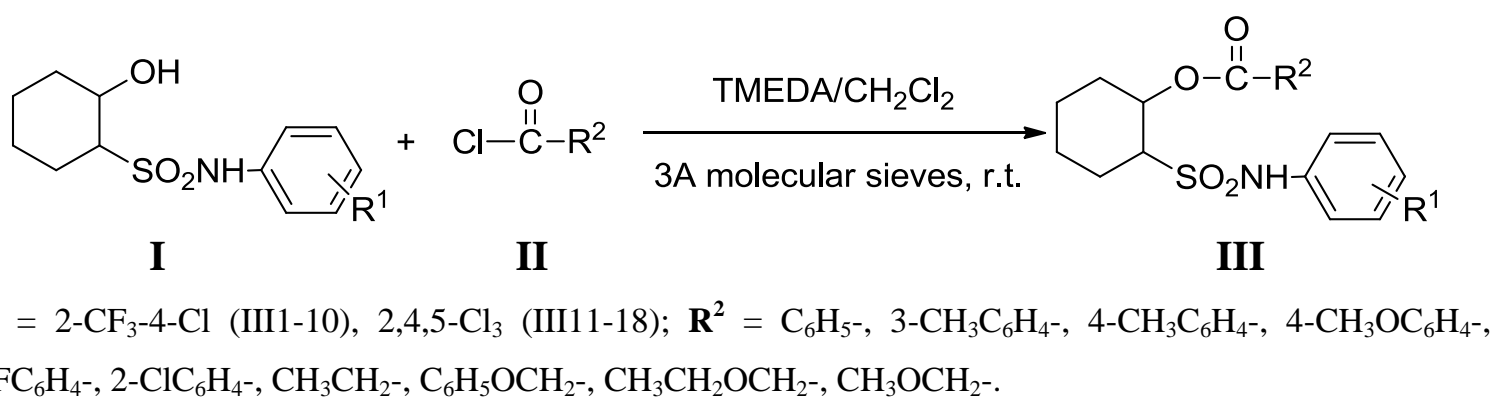

\section{Results and Discussion}

\subsection{Synthesis and Structure Elucidation}

Oxalyl chloride was used as the chlorinating agent in this experiment, which was reacted rapidly under the catalysis of DMF. The reaction made a very high yield of the acyl chloride, which was reacted with the compounds containing hydroxy group under the catalysis of TMDEA and molecular sieves to obtain a very high yield of target compounds.

In the ${ }^{1} \mathrm{H}$ NMR spectra of the compound III-5, 2- $\mathrm{H}_{\alpha}$ was split by $1-\mathrm{H}_{\alpha}, 3-\mathrm{H}_{\mathrm{e}}$ and $3-\mathrm{H}_{\alpha}$ in the cyclohexane, forming the splitting ddd peak (Figure 2). It may be the trans chair-conformation in Figure 3. Due to the directly induced effect of the sulfonyl group and the ortho-position effect of the acyloxy to the $1-\mathrm{H}_{\alpha}$, which made the $\mathrm{CH}$ signal slightly wide single peak in the spectra, the peak type was similar to that of $\mathrm{NH}$ in sulfonamide structure. In the IR spectrum, the very obvious ester carbonyl stretching vibration appeared around $1700 \mathrm{~cm}^{-1}$. NH stretching vibration was around $3200 \mathrm{~cm}^{-1}$ to $3500 \mathrm{~cm}^{-1}$. 
Figure 2. ${ }^{1} \mathrm{H}-\mathrm{NMR}$ spectrum of the compound III-5.

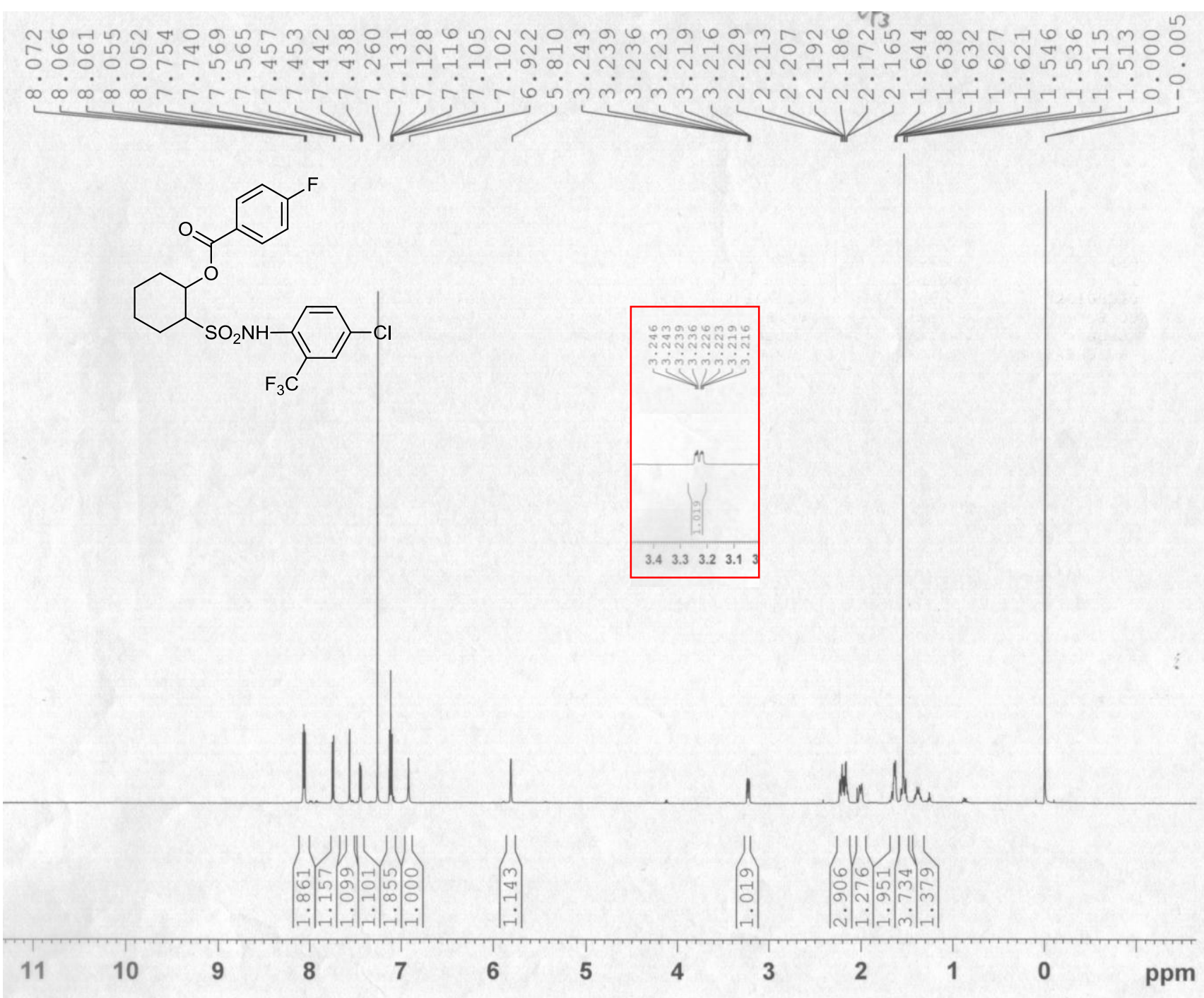

Figure 3. Proposed conformation of the compound III-5.

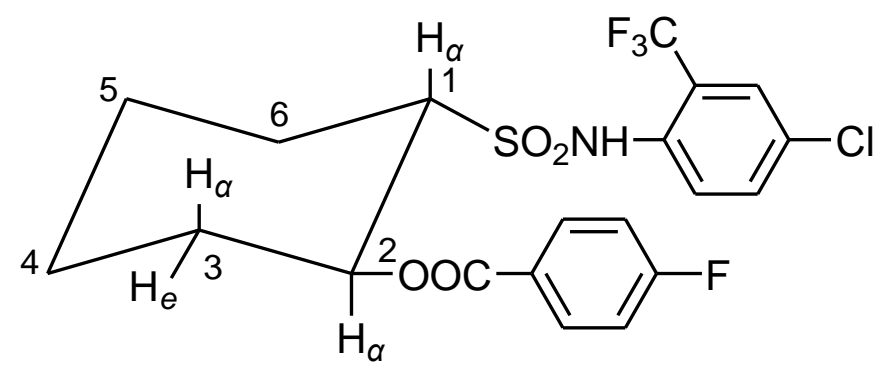

\subsection{Screening of Fungicidal Activity and SAR}

2.2.1. In Vitro Fungicidal Activity of Compounds III-1-III-18 against B. cinerea

From the results of mycelial growth rate (Table 1) and structure-activity relationship (SAR), it could be seen that acyloxy substituent showed higher antifungal activity when it was a small group. Among them, 2-substituted benzoyloxy compounds showed generally lower activity, while 
2-(2-alkoxyacetyloxy) cycloalkylsulfonamides showed good activity. The same structure-activity relationship appeared in the test of detached leaves of cucumber (Table 1). It showed obvious bioactivity when the $\mathrm{R}^{2}$ was ethoxymethyl and methoxymethyl. The bioactivity of the $N$-(2,4,5-trichlorophenyl)-2-acyloxycyclohexylsulfonamides were better than that of the $N$-(2-trifluoromethyl-4-chlorinephenyl)-2-acyloxycyclohexyl-sulfonamides. The fungicidal activity of III-18 was a little higher than that of the procymidone in vitro tests. While the detached leaves test showed that the control efficiency of III-18 was significantly higher than that of the procymidone. According to Regulation (EC) No 1107/2009, procymidone is no longer approved in the European Union. Because of the great fungicidal activity, III-18 could be a potential substitute of this fungicide.

Table 1. Fungicidal activity of compounds III-1-III-18 against $B$. cinerea.

\begin{tabular}{|c|c|c|c|}
\hline \multirow[b]{2}{*}{ Compd. } & \multicolumn{2}{|c|}{ Mycelial growth rate method } & \multirow{2}{*}{$\begin{array}{c}\text { Control efficiency on } \\
\text { detached leaves of cucumber } \\
\left(500 \mu \mathrm{g} \mathrm{mL}{ }^{-1}\right)(\%)( \pm \text { SEM })\end{array}$} \\
\hline & $\begin{array}{c}\text { EC }_{50}(95 \% \text { confidence } \\
\text { limit }) /\left(\mu \mathrm{g} \mathrm{mL}^{-1}\right)\end{array}$ & $\begin{array}{c}\mathrm{EC}_{80}(95 \% \text { confidence } \\
\text { limit }) /\left(\mu \mathrm{g} \mathrm{mL}^{-1}\right)\end{array}$ & \\
\hline III-1 & $24.36(10.23-57.99)$ & $>100$ & $61.11( \pm 5.73) \mathrm{de}$ \\
\hline III-2 & $19.94(10.51-37.80)$ & $>100$ & $27.35( \pm 2.65) \mathrm{kl}$ \\
\hline III-3 & $>100$ & $>100$ & $53.33( \pm 1.20) \mathrm{fg}$ \\
\hline III-4 & $72.28(7.10-735.67)$ & $>100$ & $18.67( \pm 3.03) \mathrm{kl}$ \\
\hline III-5 & $79.66(7.90-802.80)$ & $>100$ & $43.33( \pm 8.89)$ ghi \\
\hline III-6 & $150.33(50.71-445.65)$ & $>100$ & $58.67( \pm 6.97) \operatorname{defg}$ \\
\hline III-7 & $6.35(5.33-7.57)$ & $16.72(14.03-19.92)$ & $57.58( \pm 3.50) \mathrm{fg}$ \\
\hline III-8 & $56.23(18.13-173.76)$ & $>100$ & $48.81( \pm 9.20) \mathrm{gh}$ \\
\hline III-9 & $7.98(6.71-9.48)$ & $20.78(17.49-24.69)$ & $80.94( \pm 2.98) \mathrm{ab}$ \\
\hline III-10 & $7.46(5.75-9.69)$ & $31.31(24.13-40.68)$ & $70.67( \pm 8.70)$ cde \\
\hline III-11 & $>100$ & - & $43.60( \pm 3.25)$ hij \\
\hline III-12 & $>100$ & - & $39.74( \pm 7.32) \mathrm{jkl}$ \\
\hline III-13 & $23.86(16.65-34.19)$ & $88.48(61.75-126.78)$ & $40.47( \pm 9.55) \mathrm{ij}$ \\
\hline III-14 & $6.20(5.08-7.58)$ & $14.37(11.77-17.55)$ & $45.75( \pm 12.55) \mathrm{hi}$ \\
\hline III-15 & 29.05 (9.59-87.99) & $>100$ & $60.46( \pm 9.98)$ efg \\
\hline III-16 & $5.40(3.66-7.96)$ & $23.13(15.69-34.11)$ & $58.01( \pm 10.22) \mathrm{gh}$ \\
\hline III-17 & $16.13(11.02-23.58)$ & $92.93(63.55-135.90)$ & $50.30( \pm 6.69)$ ghi \\
\hline III-18 & $4.17(3.26-5.33)$ & $17.15(13.41-21.94)$ & $96.22( \pm 13.10) \mathrm{a}$ \\
\hline procymidone & $4.46(2.28-8.76)$ & $35.02(17.94-68.78)$ & $72.11( \pm 5.60) b c$ \\
\hline
\end{tabular}

The letters a-1 denoted the results of difference significance analysis. Means followed by the same letter within the same column are not significantly different ( $p \geq 0.05$, Fisher's LSD multiple comparison test).

\subsubsection{Fungicidal Activity of Compounds III-9 and III-18 against Ten Pathogenic Fungi}

The screening data in Table 2 indicated that the two compounds III-9 and III-18 had broad fungicidal spectra. III-9 exhibited moderate activity against ten pathogenic fungi. Compound III-18 exhibited excellent bioactivity against the most of the selected fungus species (except for the $R$. solani) and the inhibitory rate was similar to or better than the commercialized fungicide chlorothalonil. 
Table 2. Fungicidal activity of compounds III-9 and III-18 against ten pathogenic fungi.

\begin{tabular}{lccc}
\hline \multirow{2}{*}{ Fungus } & \multicolumn{2}{c}{ Inhibitory rate $\left(\mathbf{5 0} \boldsymbol{\mu g} \mathbf{~ m L}^{\mathbf{- 1}}\right)(\mathbf{\%})( \pm$ SEM $)$} \\
\cline { 2 - 4 } & III-9 & III-18 & chlorothalonil \\
\hline Curvularia lunata (Walker) Boed & $66.78( \pm 3.07)$ & $82.93( \pm 1.58)$ & $86.39( \pm 2.05)$ \\
Bipolaris maydis Shoem & $58.05( \pm 5.38)$ & $87.99( \pm 1.25)$ & $90.00( \pm 1.38)$ \\
Gibberella zeae (Schw.) Petch & $59.01( \pm 1.48)$ & $73.23( \pm 1.80)$ & $90.96( \pm 1.48)$ \\
Colletotrichum gossypii Southw & $75.53( \pm 1.87)$ & $74.42( \pm 1.01)$ & $51.73( \pm 1.57)$ \\
Sclerotinia sclerotiorum (Lib.) de Bary & $78.13( \pm 0.90)$ & $73.13( \pm 1.28)$ & $95.99( \pm 0.49)$ \\
Fusarium oxysporum & $24.02( \pm 2.13)$ & $49.77( \pm 1.40)$ & $78.07( \pm 1.35)$ \\
Macrophoma kuwatsukai Hara & $53.12( \pm 1.23)$ & $84.91( \pm 2.40)$ & $79.61( \pm 2.35)$ \\
Alternaria solani Jones et Grout & $53.76( \pm 2.30)$ & $77.03( \pm 1.45)$ & $58.94( \pm 5.17)$ \\
Corynespora cassiicola & $48.9( \pm 1.10)$ & $77.77( \pm 3.83)$ & $69.52( \pm 3.26)$ \\
Rhizoctonia solani kühn & $19.64( \pm 3.88)$ & $44.13( \pm 2.91)$ & $68.65( \pm 2.26)$ \\
\hline
\end{tabular}

\subsubsection{In Vivo Fungicidal Activity of Compounds III-18, 19 and $\mathbf{2 0}$ against $B$. cinerea}

In addition, the concentration gradient experiment was evaluated by the method of cucumber leaf to check the fungicidal activity of compounds III-18, $N$-(2,4,5-trichlorophenyl)-2hydroxycycloalkylsulfonamide (19) and $N$-(2,4,5-trichlorophenyl)-2-oxycycloalkylsulfonamide (20). The results in Table 3 showed that the activity of the compounds was gradually improved after continuous structural optimization. From the results, it could be seen that the bioactivity was not obviously different between III-18 and 19, which was much better than the activity of 20, and a little better than that of the control cyprodinil. The pathogen infection symptoms (Figure 4) showed that the leaves treated by the compound III-18 were still green and the disease spots were wet blotch plaque, which was better than the leaves treated by other compounds. The leaves treated by $\mathbf{2 0}$ and blank control were black and rotten.

Table 3. Control efficiency of III-18, 19 and 20 against $B$. cinerea (leaf method).

\begin{tabular}{|c|c|c|c|c|}
\hline $\begin{array}{l}\text { Concentration } \\
\left(\mu \mathrm{g} \mathrm{mL}^{-1}\right)\end{array}$ & Compd. & $\sim R$ & $\begin{array}{c}\text { Average diameter } \\
\text { of the spot }(\mathrm{mm})( \pm \mathrm{SEM})\end{array}$ & Inhibitory rate $(\%)$ \\
\hline \multirow{4}{*}{500} & III-18 & $-\mathrm{OCOCH}_{2} \mathrm{OC}_{2} \mathrm{H}_{5}$ & $3.0( \pm 0.6)$ & $84.12 \mathrm{a}$ \\
\hline & 19 & $-\mathrm{OH}$ & $4.3( \pm 0.5)$ & $77.40 \mathrm{a}$ \\
\hline & 20 & $=\mathrm{O}$ & $7.0( \pm 1.9)$ & $63.21 \mathrm{~b}$ \\
\hline & cyprodinil & & $4.1( \pm 0.5)$ & $78.47 \mathrm{a}$ \\
\hline \multirow{4}{*}{125} & III-18 & $-\mathrm{OCOCH}_{2} \mathrm{OC}_{2} \mathrm{H}_{5}$ & $5.4( \pm 0.6)$ & $71.50 \mathrm{a}^{\prime}$ \\
\hline & 19 & $-\mathrm{OH}$ & $6.7( \pm 0.9)$ & $64.40 \mathrm{a}^{\prime} \mathrm{b}^{\prime}$ \\
\hline & 20 & $=\mathrm{O}$ & $8.5( \pm 1.2)$ & $55.13 \mathrm{~b}^{\prime}$ \\
\hline & cyprodinil & & $7.0( \pm 0.8)$ & $62.80 \mathrm{~b}^{\prime}$ \\
\hline \multirow{4}{*}{31.25} & III-18 & $-\mathrm{OCOCH}_{2} \mathrm{OC}_{2} \mathrm{H}_{5}$ & $9.6( \pm 1.4)$ & $49.21 \mathrm{al}$ \\
\hline & 19 & $-\mathrm{OH}$ & $10.5( \pm 2.0)$ & $44.22 \mathrm{al}$ \\
\hline & 20 & $=\mathrm{O}$ & $9.1( \pm 1.8)$ & $52.02 \mathrm{al}$ \\
\hline & cyprodinil & & $8.7( \pm 0.9)$ & $54.12 \mathrm{al}$ \\
\hline 0 & CK & & $18.9( \pm 2.3)$ & 2.29 \\
\hline
\end{tabular}

The letters a-a" denoted the results of difference significance analysis. Means followed by the same letter within the same column are not significantly different ( $p \geq 0.05$, Fisher's LSD multiple comparison test). 
Figure 4. In vivo activity against $B$. cinerea in cucumber seedlings at $125 \mu \mathrm{g} \mathrm{mL}^{-1}$.

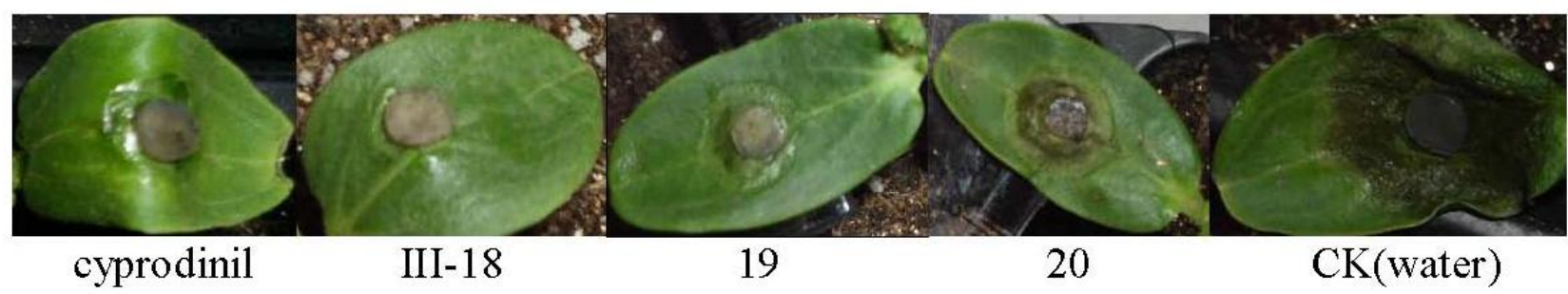

\section{Experimental Section}

\subsection{General Information}

Infrared (IR) spectra were recorded in potassium bromide disks on a Perkin Elmer Spectrum 65 spectrophotometer. Nuclear magnetic resonance (NMR) spectra were recorded in $\mathrm{CDCl}_{3}$ unless indicated otherwise with Bruker $600 \mathrm{MHz}$ or $300 \mathrm{MHz}$ spectrometers, using tetramethylsilane (TMS) as the internal standard. Elemental analysis was performed by the analytical center at the Institute of Chemistry, Chinese Academy of Sciences, Beijing, China. Melting points were measured on an X-5 melting-point apparatus, and the thermometer was uncorrected. The solvents and reagents were used as received or were dried prior to use as needed.

\subsection{Synthetic Procedures}

\subsubsection{Synthesis of Compounds I}

Compounds I were synthesized according to the method given in the references [25] and [27].

\subsubsection{Synthesis of Acyl Chloride II}

To the solution of acid $(0.05 \mathrm{~mol})$ and $N, N$-dimethyl formamide (DMF, $0.05 \mathrm{~mL}$ ) in dry dichloromethane $(20 \mathrm{~mL})$, oxalyl chloride $(0.06 \mathrm{~mol})$ was added dropwise. The mixture was stirred at 20-25 ${ }^{\circ} \mathrm{C}$ for $4 \mathrm{~h}$. And the acyl chloride was distilled under pressure.

II-1: Benzoyl chloride, colorless liquid, bp $91-92{ }^{\circ} \mathrm{C} / 31 \mathrm{mmHg}$, yield $85 \%$.

II-2: 3-Methylbenzoyl chloride, colorless liquid, bp $90-92{ }^{\circ} \mathrm{C} / 10 \mathrm{mmHg}$, yield $86 \%$.

II-3: 4-Methylbenzoyl chloride, colorless liquid, bp $117-119{ }^{\circ} \mathrm{C} / 15 \mathrm{mmHg}$, yield $88 \%$.

II-4: 4-Methoxybenzoyl chloride, white solid, bp $153-156^{\circ} \mathrm{C} / 15 \mathrm{mmHg}$, yield $80 \%$.

II-5: 4-Fluorobenzoyl chloride, colorless liquid, bp $87-88^{\circ} \mathrm{C} / 25 \mathrm{mmHg}$, yield $88 \%$.

II-6: 2-Chlorobenzoyl chloride, colorless liquid, bp $110-114{ }^{\circ} \mathrm{C} / 8 \mathrm{mmHg}$, yield $85 \%$.

II-7: Propionyl chloride, colorless, bp $77-79{ }^{\circ} \mathrm{C}$, yield $84 \%$.

II-8: Phenoxyacetyl chloride, colorless liquid, bp $103-105^{\circ} \mathrm{C} / 7 \mathrm{mmHg}$, yield $82 \%$.

II-9: 2-Ethoxyacetyl chloride, colorless liquid, bp $65-67{ }^{\circ} \mathrm{C} / 33 \mathrm{mmHg}$, yield $82 \%$.

II-10: 2-Methoxyacetyl chloride, colorless liquid, bp $112-113{ }^{\circ} \mathrm{C}$, yield $85 \%$. 


\subsubsection{Synthesis of Compounds III-1-III-18}

According to the method given in the reference [28], to the solution of $\mathbf{I}(0.01 \mathrm{~mol})$, $N, N, N^{\prime}, N^{\prime}$-tetramethylethylenediamine (TMEDA, $\left.0.006 \mathrm{~mol}\right)$ and $3 \mathrm{~A}$ molecular sieves $(2 \mathrm{~g})$ in dry dichloromethane $(30 \mathrm{~mL})$, acyl chloride II $(0.011 \mathrm{~mol})$ was added dropwise at room temperature. The mixture was stirred at room temperature for $2 \mathrm{~h}$. And the reaction was quenched by ice water $(20 \mathrm{~mL} \times 2)$, filtered and dried with anhydrous magnesium sulfate. After evaporating the solvent under vacuum, the crude product was purified by silica gel chromatography using petroleum/ethyl acetate $(10 / 1, v / v)$ as eluant to obtain III (Scheme 1).

$N$-(2-Trifluoromethyl-4-chlorophenyl)-2-benzoyloxy-cyclohexylsulfonamide (III-1). White crystal, yield 90\%, m.p. $119-121{ }^{\circ} \mathrm{C},{ }^{1} \mathrm{H}-\mathrm{NMR}\left(\mathrm{CDCl}_{3}, 300 \mathrm{MHz}\right.$, Figure $\left.\mathrm{S} 1\right) \delta$ : $1.37-2.25\left(\mathrm{~m}, 8 \mathrm{H}, 4 \mathrm{CH}_{2}\right), 3.22-3.28$ $(\mathrm{m}, 1 \mathrm{H}, \mathrm{CH}-\mathrm{O}), 5.84\left(\mathrm{~s}, 1 \mathrm{H}, \mathrm{CH}-\mathrm{SO}_{2}\right), 6.98(\mathrm{~s}, 1 \mathrm{H}, \mathrm{NH}), 7.38-8.06\left(\mathrm{~m}, 8 \mathrm{H}, \mathrm{C}_{6} \mathrm{H}_{3}+\mathrm{C}_{6} \mathrm{H}_{5}\right) ; \mathrm{IR}(\mathrm{KBr})$ v: 3273, 2944, 2858, $1717 \mathrm{~cm}^{-1}$. Anal calcd. for $\mathrm{C}_{20} \mathrm{H}_{19} \mathrm{ClF}_{3} \mathrm{NO}_{4} \mathrm{~S}: \mathrm{C} 52.01, \mathrm{H} 4.15, \mathrm{~N} 3.03$; Found: C 51.82, H 4.13, N, 3.21.

$N$-(2-Trifluoromethyl-4-chlorophenyl)-2-(3-methyl-benzoyloxy)-cyclohexylsulfonamide (III-2). White crystal, yield 88\%, m.p. $112-114{ }^{\circ} \mathrm{C},{ }^{1} \mathrm{H}-\mathrm{NMR}\left(\mathrm{CDCl}_{3}, 300 \mathrm{MHz}\right.$, Figure S2) $\delta: 1.41-2.25$ (m, 8H, $\left.4 \mathrm{CH}_{2}\right), 2.40\left(\mathrm{~s}, 3 \mathrm{H}, \mathrm{CH}_{3}\right), 3.22-3.27(\mathrm{~m}, 1 \mathrm{H}, \mathrm{CH}-\mathrm{O}), 5.83\left(\mathrm{~s}, 1 \mathrm{H}, \mathrm{CH}-\mathrm{SO}_{2}\right), 6.98(\mathrm{~s}, 1 \mathrm{H}, \mathrm{NH})$, 7.29-7.83 (m, 7H, $\left.\mathrm{C}_{6} \mathrm{H}_{3}+\mathrm{C}_{6} \mathrm{H}_{4}\right)$; $\mathrm{IR}(\mathrm{KBr}) v$ : 3283, 2939, 2855, $1720 \mathrm{~cm}^{-1}$. Anal calcd. for $\mathrm{C}_{21} \mathrm{H}_{21} \mathrm{ClF}_{3} \mathrm{NO}_{4} \mathrm{~S}$ : C 53.00, H 4.45, N 2.94; Found: C 53.41, H 4.28, N 2.96.

$N$-(2-Trifluoromethyl-4-chlorophenyl)-2-(4-methyl-benzoyloxy)-cyclohexylsulfonamide (III-3). White crystal, yield 87\%, m.p. $128-130{ }^{\circ} \mathrm{C},{ }^{1} \mathrm{H}-\mathrm{NMR}\left(\mathrm{CDCl}_{3}, 600 \mathrm{MHz}\right.$, Figure S3) $\delta: 1.37-2.23$ $\left(\mathrm{m}, 8 \mathrm{H}, 4 \mathrm{CH}_{2}\right), 2.40\left(\mathrm{~s}, 3 \mathrm{H}, \mathrm{CH}_{3}\right), 3.22-3.24(\mathrm{~m}, 1 \mathrm{H}, \mathrm{CH}-\mathrm{O}), 5.81\left(\mathrm{~s}, 1 \mathrm{H}, \mathrm{CH}-\mathrm{SO}_{2}\right), 6.95(\mathrm{~s}, 1 \mathrm{H}, \mathrm{NH})$, 7.22-7.91 (m, 7H, $\left.\mathrm{C}_{6} \mathrm{H}_{3}+\mathrm{C}_{6} \mathrm{H}_{4}\right)$; $\mathrm{IR}(\mathrm{KBr}) v$ : 3277, 2949, 2865, $1708 \mathrm{~cm}^{-1}$. Anal calcd. for $\mathrm{C}_{21} \mathrm{H}_{21} \mathrm{ClF}_{3} \mathrm{NO}_{4} \mathrm{~S}$ : C 53.00, H 4.45, N 2.94; Found: C 52.97, H 4.50, N 2.91.

$N$-(2-Trifluoromethyl-4-chlorophenyl)-2-(4-methoxy-benzoyloxy)-cyclohexylsulfonamide (III-4). White crystal, yield 83\%, m.p. 94-96 ${ }^{\circ} \mathrm{C},{ }^{1} \mathrm{H}-\mathrm{NMR}\left(\mathrm{CDCl}_{3}, 600 \mathrm{MHz}\right.$, Figure S4) $\delta: 1.37-2.22$ $\left(\mathrm{m}, 8 \mathrm{H} 4 \mathrm{CH}_{2}\right), 3.21-3.24(\mathrm{~m}, 1 \mathrm{H} \mathrm{CH}-\mathrm{O}), 3.86\left(\mathrm{~s}, 3 \mathrm{H} \mathrm{OCH}_{3}\right), 5.79\left(\mathrm{~s}, 1 \mathrm{H} \mathrm{CH}-\mathrm{SO}_{2}\right), 6.98(\mathrm{~s}, 1 \mathrm{H} \mathrm{NH})$, 6.90-7.99 (m, 7H C $\left.\mathrm{C}_{6} \mathrm{H}_{3}+\mathrm{C}_{6} \mathrm{H}_{4}\right)$; $\mathrm{IR}(\mathrm{KBr}) v$ : 3277, 2949, 2865, $1708 \mathrm{~cm}^{-1}$. Anal calcd. for $\mathrm{C}_{21} \mathrm{H}_{21} \mathrm{ClF}_{3} \mathrm{NO}_{5} \mathrm{~S}$ : C 51.27; H 4.30, N 2.85; Found: C 51.21; H 4.19, N 2.93.

$N$-(2-Trifluoromethyl-4-chlorophenyl)-2-(4-fluoro-benzoyloxy)-cyclohexylsulfonamide

(III-5).

White crystal, yield 89\%, m.p. 120-122 ${ }^{\circ} \mathrm{C},{ }^{1} \mathrm{H}-\mathrm{NMR}\left(\mathrm{CDCl}_{3}, 600 \mathrm{MHz}\right.$, Figure S5) $\delta$ : 1.37-2.22 $\left(\mathrm{m}, 8 \mathrm{H}, 4 \mathrm{CH}_{2}\right), 3.23(\mathrm{ddd}, J=12,4.2,1.8 \mathrm{~Hz}, 1 \mathrm{H}, \mathrm{CH}-\mathrm{O}), 5.81\left(\mathrm{~s}, 1 \mathrm{H}, \mathrm{CH}-\mathrm{SO}_{2}\right), 6.92(\mathrm{~s}, 1 \mathrm{H}, \mathrm{NH})$, 7.09-8.07 (m, 7H, $\left.\mathrm{C}_{6} \mathrm{H}_{3}+\mathrm{C}_{6} \mathrm{H}_{4}\right)$; IR(KBr) v: 3277, 2935, 2859, $1760 \mathrm{~cm}^{-1}$. Anal calcd. for $\mathrm{C}_{20} \mathrm{H}_{18} \mathrm{ClF}_{4} \mathrm{NO}_{4} \mathrm{~S}$ : C 50.06, H 3.78, N 2.92; Found: C 50.05, H 3.89, N 2.79.

$N$-(2-Trifluoromethyl-4-chlorophenyl)-2-(2-chloro-benzoyloxy)-cyclohexylsulfonamide

(III-6).

White crystal, yield 86\%, m.p. $98-100{ }^{\circ} \mathrm{C},{ }^{1} \mathrm{H}-\mathrm{NMR}\left(\mathrm{CDCl}_{3}, 600 \mathrm{MHz}\right.$, Figure S6) $\delta$ : 1.37-2.25 $\left(\mathrm{m}, 8 \mathrm{H}, 4 \mathrm{CH}_{2}\right), 3.21-3.24(\mathrm{~m}, 1 \mathrm{H}, \mathrm{CH}-\mathrm{O}), 5.81\left(\mathrm{~s}, 1 \mathrm{H}, \mathrm{CH}-\mathrm{SO}_{2}\right), 6.96(\mathrm{~s}, 1 \mathrm{H}, \mathrm{NH}), 7.31-7.93$ 
(m, 7H, $\left.\mathrm{C}_{6} \mathrm{H}_{3}+\mathrm{C}_{6} \mathrm{H}_{4}\right)$; IR(KBr) v: 3247, 2954, 2869, $1731 \mathrm{~cm}^{-1}$. Anal calcd. for $\mathrm{C}_{20} \mathrm{H}_{18} \mathrm{Cl}_{2} \mathrm{~F}_{3} \mathrm{NO}_{4} \mathrm{~S}$ : C 48.40, H 3.66, N 2.82; Found: C 48.20, H 3.45, N 2.92.

$N$-(2-Trifluoromethyl-4-chlorophenyl)-2-propionyloxy-cyclohexylsulfonamide (III-7). Oil, yield 73\%, ${ }^{1} \mathrm{H}-\mathrm{NMR}\left(\mathrm{CDCl}_{3}, 600 \mathrm{MHz}\right.$, Figure S7) $\delta: 1.13-2.41\left(\mathrm{~m}, 13 \mathrm{H}, 4 \mathrm{CH}_{2}+\mathrm{C}_{2} \mathrm{H}_{5}\right), 3.08-3.11(\mathrm{~m}, 1 \mathrm{H}, \mathrm{CH}-\mathrm{O})$, $5.66\left(\mathrm{~s}, 1 \mathrm{H}, \mathrm{CH}-\mathrm{SO}_{2}\right), 7.06(\mathrm{~s}, 1 \mathrm{H}, \mathrm{NH}), 7.49-7.72\left(\mathrm{~m}, 3 \mathrm{H}, \mathrm{C}_{6} \mathrm{H}_{3}\right)$; IR(KBr) v: 3276, 2959, 2859, $1752 \mathrm{~cm}^{-1}$. Anal calcd. for $\mathrm{C}_{16} \mathrm{H}_{19} \mathrm{ClF}_{3} \mathrm{NO}_{4} \mathrm{~S}$ : C 46.44, H 4.63, N 3.38; Found: C 46.22, H 4.43, N 3.54.

$N$-(2-Trifluoromethyl-4-chlorophenyl)-2-phenoxyacetoxy-cyclohexylsulfonamide (III-8). Oil, yield 78\%, ${ }^{1} \mathrm{H}-\mathrm{NMR}\left(\mathrm{CDCl}_{3}, 600 \mathrm{MHz}\right.$, Figure S8) $\delta: 1.13-2.05\left(\mathrm{~m}, 8 \mathrm{H}, 4 \mathrm{CH}_{2}\right), 3.09-3.12(\mathrm{~m}, 1 \mathrm{H}, \mathrm{CH}-\mathrm{O}), 4.70$ (s, 2H, $\mathrm{CH}_{2}-\mathrm{O}$ ), 5.77 (s, 1H, CH-SO $\mathrm{S}_{2}$ ), 6.88-7.71 (m, 9H, $\left.\mathrm{C}_{6} \mathrm{H}_{3}+\mathrm{C}_{6} \mathrm{H}_{5}+\mathrm{NH}\right)$; IR(KBr) v: 3278, 2944, 2864, $1754 \mathrm{~cm}^{-1}$. Anal calcd. for $\mathrm{C}_{21} \mathrm{H}_{21} \mathrm{ClF}_{3} \mathrm{NO}_{5} \mathrm{~S}$ : C 51.27, H 4.30, N 2.85; Found: C 51.43, H 4.38, N 2.65 .

$\mathrm{N}$-(2-Trifluoromethyl-4-chlorophenyl)-2-(2-ethoxyacetoxy)-cyclohexylsulfonamide (III-9). White crystal, yield 79\%, m.p. 85-87 ${ }^{\circ} \mathrm{C},{ }^{1} \mathrm{H}-\mathrm{NMR}\left(\mathrm{CDCl}_{3}, 600 \mathrm{MHz}\right.$, Figure S9) $\delta: 1.22-2.09\left(\mathrm{~m}, 11 \mathrm{H}, 4 \mathrm{CH}_{2}+\mathrm{CH}_{3}\right)$, $3.10-3.13(\mathrm{~m}, 1 \mathrm{H}, \mathrm{CH}-\mathrm{O}), 3.62\left(\mathrm{q}, J=7.2 \mathrm{~Hz}, 2 \mathrm{H}, \mathrm{CH}_{2} \mathrm{O}\right), 4.12\left(\mathrm{~s}, 2 \mathrm{H}, \mathrm{OCH}_{2} \mathrm{CO}\right), 5.74$ (s, $\left.1 \mathrm{H}, \mathrm{CH}-\mathrm{SO}_{2}\right), 7.02(\mathrm{~s}, 1 \mathrm{H}, \mathrm{NH}), 7.50-7.71\left(\mathrm{~m}, 3 \mathrm{H}, \mathrm{C}_{6} \mathrm{H}_{3}\right) ; \mathrm{IR}(\mathrm{KBr})$ v: 3282, 2959, 2859, $1760 \mathrm{~cm}^{-1}$. Anal calcd. for $\mathrm{C}_{17} \mathrm{H}_{21} \mathrm{ClF}_{3} \mathrm{NO}_{5} \mathrm{~S}$ : C 46.00, H 4.77, N 3.16; Found: C 45.87, H 4.79, N 3.32.

$N$-(2-Trifluoromethyl-4-chlorophenyl)-2-(2-methoxyacetoxy)-cyclohexylsulfonamide (III-10). White crystal, yield 83\%, m.p. 94-96 ${ }^{\circ} \mathrm{C},{ }^{1} \mathrm{H}-\mathrm{NMR}\left(\mathrm{CDCl}_{3}, 300 \mathrm{MHz}\right.$, Figure $\left.\mathrm{S} 10\right)$ ) : 1.33-2.08 (m, 8H, $4 \mathrm{CH}_{2}$ ), 3.09-3.15 (m, 1H, CH-O), 3.47 (s, 3H, CH 3 O), 4.08 (s, 2H, OCH $\mathrm{OCO}_{2}$ ), 5.75 (s, $\left.1 \mathrm{H}, \mathrm{CH}-\mathrm{SO}_{2}\right)$, $7.03(\mathrm{~s}, 1 \mathrm{H}, \mathrm{NH}), 7.49-7.72\left(\mathrm{~m}, 3 \mathrm{H}, \mathrm{C}_{6} \mathrm{H}_{3}\right)$; IR(KBr) v: 3278, 2953, 2860, $1751 \mathrm{~cm}^{-1}$. Anal calcd. for $\mathrm{C}_{16} \mathrm{H}_{19} \mathrm{ClF}_{3} \mathrm{NO}_{5} \mathrm{~S}$ : C 44.71, H 4.46, N 3.26; Found: C 44.55, H 4.52, N 3.19.

$N$-(2,4,5-Trichlorophenyl)-2-benzoyloxy-cyclohexylsulfonamide (III-11). White crystal, yield 76\%, m.p. ${ }^{179-181 ~}{ }^{\circ} \mathrm{C},{ }^{1} \mathrm{H}-\mathrm{NMR}\left(\mathrm{CDCl}_{3}, 300 \mathrm{MHz}\right.$, Figure S11) $\delta(\mathrm{ppm}): 1.41-2.27\left(\mathrm{~m}, 8 \mathrm{H}, 4 \mathrm{CH}_{2}\right)$, 3.22-3.28 (m, 1H, CH-O), $5.83\left(\mathrm{~s}, 1 \mathrm{H}, \mathrm{CH}-\mathrm{SO}_{2}\right), 7.39-8.07\left(\mathrm{~m}, 8 \mathrm{H}, \mathrm{C}_{6} \mathrm{H}_{2}+\mathrm{C}_{6} \mathrm{H}_{5}+\mathrm{NH}\right)$; IR(KBr) v: 3236, 2946, 2859, $1761 \mathrm{~cm}^{-1}$. Anal calcd. for $\mathrm{C}_{19} \mathrm{H}_{18} \mathrm{Cl}_{3} \mathrm{NO}_{4} \mathrm{~S}: \mathrm{C} 49.31, \mathrm{H} 3.92, \mathrm{~N} 3.03$; Found: C 49.69, H 3.79, N 3.05.

$N$-(2,4,5-Trichlorophenyl)-2-(3-methylbenzoyloxy)-cyclohexylsulfonamide (III-12). White crystal, yield $78 \%$, m.p. $176-178{ }^{\circ} \mathrm{C},{ }^{1} \mathrm{H}-\mathrm{NMR}\left(\mathrm{CDCl}_{3}, 300 \mathrm{MHz}\right.$, Figure $\left.\mathrm{S} 12\right) \delta: 1.45-2.27\left(\mathrm{~m}, 8 \mathrm{H}, 4 \mathrm{CH}_{2}\right), 2.41$ (s, 3H, CH 3 ), 3.18-3.24 (m, 1H, CH-O), 5.83 (s, 1H, CH-SO $), 7.20$ (s, 1H, NH), 7.29-7.83 (m, 6H, $\left.\mathrm{C}_{6} \mathrm{H}_{2}+\mathrm{C}_{6} \mathrm{H}_{4}\right)$; IR(KBr) v: 3280, 2940, 2854, $1754 \mathrm{~cm}^{-1}$. Anal calcd. for $\mathrm{C}_{20} \mathrm{H}_{20} \mathrm{Cl}_{3} \mathrm{NO}_{4} \mathrm{~S}: \mathrm{C} 50.38$, H 4.23, N 2.94; Found: C 50.25, H 4.08, N 2.99.

$N$-(2,4,5-Trichlorophenyl)-2-(4-methylbenzoyloxy)-cyclohexylsulfonamide (III-13). White crystal, yield $78 \%$, m.p. $170-172{ }^{\circ} \mathrm{C},{ }^{1} \mathrm{H}-\mathrm{NMR}\left(\mathrm{CDCl}_{3}, 600 \mathrm{MHz}\right.$, Figure $\left.\mathrm{S} 13\right) \delta: 1.42-2.37\left(\mathrm{~m}, 8 \mathrm{H}, 4 \mathrm{CH}_{2}\right), 2.41$ (s, 3H, $\mathrm{CH}_{3}$ ), 3.19-3.22 (m, 1H, CH-O), 5.83 (d,d,d, $\left.J=11.70,3.90,1.80 \mathrm{~Hz}, 1 \mathrm{H}, \mathrm{CH}-\mathrm{SO}_{2}\right), 7.18$ (s, 1H, NH), 7.30-7.83 (m, 6H, $\left.\mathrm{C}_{6} \mathrm{H}_{2}+\mathrm{C}_{6} \mathrm{H}_{4}\right)$; IR(KBr) v: 3278, 2946, 2859, $1708 \mathrm{~cm}^{-1}$. Anal calcd. for $\mathrm{C}_{20} \mathrm{H}_{20} \mathrm{Cl}_{3} \mathrm{NO}_{4} \mathrm{~S}$ : C 50.38, H 4.23, N 2.94; Found: C 50.63, H 4.11, N 2.97. 
$N$-(2,4,5-Trichlorophenyl)-2-(4-fluorobenzoyloxy)-cyclohexylsulfonamide (III-14). White crystal, yield 79\%, m.p. ${ }^{160-161 ~}{ }^{\circ} \mathrm{C},{ }^{1} \mathrm{H}-\mathrm{NMR}\left(\mathrm{CDCl}_{3}, 300 \mathrm{MHz}\right.$, Figure S14) $\delta: 1.49-2.25\left(\mathrm{~m}, 8 \mathrm{H}, 4 \mathrm{CH}_{2}\right)$, 3.15-3.20 (m, 1H, CH-O), 5.89 (s, 1H, CH-SO ${ }_{2}$ ), 7.20 (s, 1H, NH), 7.29-7.90 (m, 6H, $\left.\mathrm{C}_{6} \mathrm{H}_{2}+\mathrm{C}_{6} \mathrm{H}_{4}\right)$; IR(KBr) v: 3278, 2940, 2859, $1708 \mathrm{~cm}^{-1}$. Anal calcd. for $\mathrm{C}_{19} \mathrm{H}_{17} \mathrm{Cl}_{3} \mathrm{FNO}_{4} \mathrm{~S}: \mathrm{C} 47.47, \mathrm{H} 3.56, \mathrm{~N} 2.91$; Found: C 46.99, H 3.45, N 2.97.

$N$-(2,4,5-Trichlorophenyl)-2-(2-chlorobenzoyloxy)-cyclohexylsulfonamide (III-15). White crystal, yield 75\%, m.p. ${ }^{145-147}{ }^{\circ} \mathrm{C},{ }^{1} \mathrm{H}-\mathrm{NMR}\left(\mathrm{CDCl}_{3}, 300 \mathrm{MHz}\right.$, Figure $\left.\mathrm{S} 15\right) \delta: 1.43-2.25\left(\mathrm{~m}, 8 \mathrm{H}, 4 \mathrm{CH}_{2}\right)$, 3.16-3.21 (m, 1H, CH-O), $5.81\left(\mathrm{~s}, 1 \mathrm{H}, \mathrm{CH}-\mathrm{SO}_{2}\right), 7.94-8.90\left(\mathrm{~m}, 7 \mathrm{H}, \mathrm{C}_{6} \mathrm{H}_{2}+\mathrm{C}_{6} \mathrm{H}_{4}+\mathrm{NH}\right)$; $\mathrm{IR}(\mathrm{KBr})$ v: 3244, 2946, 2859, $1708 \mathrm{~cm}^{-1}$. Anal calcd. for $\mathrm{C}_{19} \mathrm{H}_{17} \mathrm{Cl}_{4} \mathrm{NO}_{4} \mathrm{~S}$ : C 45.90, H 3.45, N 2.82; Found: C 46.23, H 3.44, N 2.76 .

$N$-(2,4,5-Trichlorophenyl)-2-propionyloxy-cyclohexylsulfonamide (III-16). White crystal, yield 56\%, m.p. ${ }^{130-131 ~}{ }^{\circ} \mathrm{C},{ }^{1} \mathrm{H}-\mathrm{NMR}\left(\mathrm{CDCl}_{3}, 600 \mathrm{MHz}\right.$, Figure S16) $\delta: 1.17-2.43\left(\mathrm{~m}, 13 \mathrm{H}, 4 \mathrm{CH}_{2}+\mathrm{C}_{2} \mathrm{H}_{5}\right)$, 3.01-3.04 (m, 1H, CH-O), 5.65 (s, 1H, CH-SO $)_{2}, 7.32$ (s, 1H, NH), 7.50-7.82 (m, 2H, $\left.\mathrm{C}_{6} \mathrm{H}_{2}\right)$; $\mathrm{IR}(\mathrm{KBr})$ v: 3251, 2940, 2859, $1734 \mathrm{~cm}^{-1}$. Anal calcd. for $\mathrm{C}_{15} \mathrm{H}_{18} \mathrm{Cl}_{3} \mathrm{NO}_{4} \mathrm{~S}: \mathrm{C} 43.44, \mathrm{H} \mathrm{4.37,} \mathrm{N} \mathrm{3.38;} \mathrm{Found:} \mathrm{C}$ 43.55, H 4.49, N 3.31 .

$N$-(2,4,5-Trichlorophenyl)-2-phenoxyacetoxy-cyclohexylsulfonamide (III-17). White crystal, yield $55 \%$, m.p. $146-148{ }^{\circ} \mathrm{C},{ }^{1} \mathrm{H}-\mathrm{NMR}\left(\mathrm{CDCl}_{3}, 600 \mathrm{MHz}\right.$, Figure $\left.\mathrm{S} 17\right), \delta(\mathrm{ppm}): 1.31-2.13\left(\mathrm{~m}, 8 \mathrm{H}, 4 \mathrm{CH}_{2}\right)$, 3.02-3.06 (m, 1H, CH-O), $4.71\left(\mathrm{~s}, 2 \mathrm{H}, \mathrm{CH}_{2}-\mathrm{O}\right), 5.77\left(\mathrm{~s}, 1 \mathrm{H}, \mathrm{CH}-\mathrm{SO}_{2}\right), 6.95-7.83(\mathrm{~m}, 8 \mathrm{H}$, $\left.\mathrm{C}_{6} \mathrm{H}_{2}+\mathrm{C}_{6} \mathrm{H}_{5}+\mathrm{NH}\right)$; IR(KBr) v: 3317, 2940, 2859, $1728 \mathrm{~cm}^{-1}$. Anal calcd. for $\mathrm{C}_{20} \mathrm{H}_{20} \mathrm{Cl}_{3} \mathrm{NO}_{5} \mathrm{~S}$ : C 48.74, H 4.09, N 2.84; Found: C 48.66, H 4.01, N 2.98.

$N$-(2,4,5-Trichlorophenyl)-2-(2-ethoxyacetoxy)-cyclohexylsulfonamide (III-18). White crystal, yield $43 \%$, m.p. $124-126{ }^{\circ} \mathrm{C},{ }^{1} \mathrm{H}-\mathrm{NMR}\left(\mathrm{CDCl}_{3}, 600 \mathrm{MHz}\right.$, Figure S18) $\delta: 1.24-2.12\left(\mathrm{~m}, 11 \mathrm{H}, 4 \mathrm{CH}_{2}+\mathrm{CH}_{3}\right)$, 3.03-3.06 (m, 1H, CH-O), $3.63\left(\mathrm{q}, J=7.2 \mathrm{~Hz}, 2 \mathrm{H}, \mathrm{CH}_{2} \mathrm{O}\right), 4.15\left(\mathrm{~s}, 2 \mathrm{H}, \mathrm{OCH}_{2} \mathrm{CO}\right), 5.73(\mathrm{~s}, 1 \mathrm{H}$, $\left.\mathrm{CH}-\mathrm{SO}_{2}\right), 7.34$ (s, 1H, NH), 7.49-7.84 (m, 2H, $\left.\mathrm{C}_{6} \mathrm{H}_{2}\right)$; IR(KBr) v: 3336, 2940, 2859, $1754 \mathrm{~cm}^{-1}$. Anal calcd. for $\mathrm{C}_{16} \mathrm{H}_{20} \mathrm{Cl}_{3} \mathrm{NO}_{5} \mathrm{~S}$ : C 43.21, H 4.53, N 3.15; Found: C 42.86, H 4.46, N 3.21.

\subsection{Bioassay of Fungicidal Activity}

\subsubsection{Effect of the Title Compounds on the Mycelial Growth}

Fungicidal activities of the compounds against $B$. cinerea were evaluated using the method given in reference [27]. The title compounds III were dissolved in acetone and mixed with sterile molten potato dextrose agar (PDA) to obtain the gradient concentrations of 50, 25, 12.5, 6.25, 3.13, 1.56 and $0.78 \mu \mathrm{g} \mathrm{mL}^{-1}$. The commercial fungicide procymidone (with a percentage composition of 96\%) was used as the positive control. The inhibition rate was calculated according to Equation (1). The $\mathrm{EC}_{50}$ and $\mathrm{EC}_{80}$ values of compounds III were estimated using logit analysis, and the results are given in Table 1.

$$
I_{1}=\left(\bar{D}_{1}-\bar{D}_{0}\right) / \bar{D}_{1} \times 100 \%
$$

where $I_{1}$ is the inhibition rate, $D_{1}$ is the average diameter of mycelia in the blank test, and $D_{0}$ is the average diameter of mycelia in the presence of compounds. 


\subsubsection{Effect of Compounds III-9 and III-18 against Ten Major Crop Diseases}

The fungicidal activity of compounds III-9 and III-18 against ten kinds of fungi was assessed using the mycelium growth test on PDA. The compounds were dissolved in acetone and mixed PDA to obtain final concentration of $50 \mu \mathrm{g} \mathrm{mL}^{-1}$. Chlorothalonil was used as the positive control. Other test conditions were the same with the method given in the mycelial growth, and the results were shown in Table 2.

\subsubsection{Effect on the Ability of B. cinerea to Colonize Detached Leaves of Cucumber}

The compounds III-1-III-18 were confected to 2.5\% EC formulations, which were diluted to concentration of $500 \mu \mathrm{g} \mathrm{mL}^{-1}$ with water to obtain the solutions that were spread on the surface of the cucumber leaves. After air drying for $2 \mathrm{~h}$ at $23{ }^{\circ} \mathrm{C}$, the upper sides of the leaves were inoculated with $5 \mathrm{~mm}$ plugs of $B$. cinerea, which was maintained on PDA [25]. The cucumber leaves were maintained at $24 \pm 1{ }^{\circ} \mathrm{C}$ in culture dishes. The inhibition rate was calculated and the results were shown in Table 1 .

\subsubsection{In Vivo Fungicidal Activity against B. cinerea by Pot Culture Test Method in Greenhouse}

Using pot culture test method according to the reference [25], the in vivo fungicidal activity of the title compounds against $B$. cinerea was evaluated in greenhouse. $B$. cinerea was maintained on potato dextrose agar (PDA) medium at $4{ }^{\circ} \mathrm{C}$. The culture plates were cultivated at $24 \pm 1{ }^{\circ} \mathrm{C}$. Germination was conducted by soaking cucumber seeds in water for $2 \mathrm{~h}$ at $50{ }^{\circ} \mathrm{C}$ and then keeping the seeds moist for $24 \mathrm{~h}$ at $28{ }^{\circ} \mathrm{C}$ in an incubator. When the radicles were $0.5 \mathrm{~cm}$, the seeds were grown in plastic pots containing a 1:1 $(\mathrm{v} / \mathrm{v})$ mixture of vermiculite and peat. Cucumber plants used for inoculations were at the stage of two seed leaves. The compounds III-18, 19 and 20 were confected to $2.5 \%$ EC formulations, which were diluted to concentration of 500, 125 and $31.25 \mu \mathrm{g} \mathrm{mL}^{-1}$ with water to obtain the solutions. Tested compounds and commercial fungicides were sprayed with a hand spray on the surface of the seed leaves. Water sprayed seed leaves were set as the CK (Figure 4). After drying, the upper sides of the leaves were inoculated with $5 \mathrm{~mm}$ plugs of $B$. cinerea, which was maintained on PDA [25]. The plants were maintained at $24 \pm 1{ }^{\circ} \mathrm{C}$ and above $80 \%$ relative humidity in greenhouse. The fungicidal activity was evaluated and the results were shown in Table 3 and Figure 4.

\section{Conclusions}

$N$-substituted phenyl-2-acyloxycyclohexylsulfonamides were designed and synthesized. Their structures were confirmed by ${ }^{1} \mathrm{H}$ NMR, IR and elementary analysis. Their fungicidal activity was better than the leading compounds $N$-substituted phenyl-2-hydroxycycloalkylsulfonamides. Among them, compound III-18 showed better in vitro and in vivo fungicidal activity than the fungicides procymidone and cyprodinil. In addition, this new compound had broader fungicidal spectra than chlorothalonil. The preliminary structure and activity relationship showed that the 2-alkoxyacetoxy groups were considerably more likely to improve the activity of these compounds. 


\section{Acknowledgments}

This work was supported by the National Natural Science Foundation of China (31101466 and 21102173), and Program for Liaoning Excellent Talents in University (LJQ2012059).

\section{Conflicts of Interest}

The authors declare no conflict of interest.

\section{References}

1. Pierre, B.; Martine, V.L.; Arlette, N.; Jean-Michel, L.; Joseph, V.; Stéphane, L.; Gérard, D.; Alain, N. New 2-sulfonamidothiazoles substituted at C-4: Synthesis of polyoxygenated aryl derivatives and in vitro evaluation of antifungal activity. Eur. J. Med. Chem. 1999, 34, 773-779.

2. Ashis, K.; Saha, L.L.; Patrick, M.; Frank, O. Novel antifungals based on 4-substituted imidazole: Solid-phase synthesis of substituted aryl sulfonamides towards optimization of in vitro activity. Bioorg. Med. Chem. Lett. 2000, 10, 2735-2739.

3. Iraj, R.E.; Charalabos, C.; Panagiotis, Z.; Athina, G.; Marina, S.; Jasmina, G.; Ana, C. Sulfonamide-1,2,4-triazole derivatives as antifungal and antibacterial agents: Synthesis, biological evaluation, lipophilicity, and conformational studies. Bioorg. Med. Chem. 2008, 16, 1150-1161.

4. Mahantesha, B.K.; Shivashankar, M.V.; Kulkarni, V.P.; Rasal, H.; Patel, S.S.; Mutha, A.A.M. Synthesis and antimicrobial studies on novel sulfonamides containing 4-azidomethyl coumarin. Eur. J. Med. Chem. 2010, 45, 1151-1157.

5. Zahid, H.; Chohan, M.H.; Youssoufi, Aliasghar, J.; Taibi, B.H. Identification of antibacterial and antifungal pharmacophore sites for potent bacteria and fungi inhibition: Indolenyl sulfonamide derivatives. Eur. J. Med. Chem. 2010, 45, 1189-1199.

6. Zoumpoulakis, P.; Camoutsis, C.; Pairas, G.; Soković, M.; Glamočlija, J.; Potamitis, C.; Pitsas, A. Synthesis of novel sulfonamide-1,2,4-triazoles, 1,3,4-thiadiazoles and 1,3,4-oxadiazoles, as potential antibacterial and antifungal agents. Biological evaluation and conformational analysis studies. Eur. J. Med. Chem. 2012, 20, 1569-1583.

7. Keche, A.P.; Hatnapure, G.D.; Tale, R.H.; Rodge, A.H.; Birajdar, S.S.; Kamble, V.M. A novel pyrimidine derivatives with aryl urea, thiourea and sulfonamide moieties: Synthesis, anti-inflammatory and antimicrobial evaluation. Bioorg. Med. Chem. Lett. 2012, 22, 3445-3448.

8. Keche, A.P.; Hatnapure, G.D.; Tale, R.H.; Rodge, A.H.; Kamble, V.M. Synthesis, anti-inflammatory and antimicrobial evaluation of novel 1-acetyl-3,5-diaryl-4,5-dihydro (1H) pyrazole derivatives bearing urea, thiourea and sulfonamide moieties. Bioorg. Med. Chem. Lett. 2012, 22, 6611-6615.

9. Fujita, T. Nebijin (flusulfamide, MTF-651), a new soil fungicide. Agrochem. Jpn. 1994, 65, 17-19.

10. Tanaka, S.; Kochi, S.I.; Kunita, H.; Ito, S.I.; Kameya-Iwaki, M. Biological mode of action of the fungicide, Flusulfamide, against Plasmodiophora brassicae (clubroot). Eur. J. Plant. Pathol. 1999, $105,577-584$. 
11. Tabuchi, T.; Yamamoto, T.; Nakayama, M. Preparation of Aryl- or Heterocyclyl-Sulfonamide Derivatives as Agricultural and Horticultural Microbicides. WO Patent WO 2,000,065,913, 9 November 2000.

12. Shigeru, M.; Satoshi, A.; Tomona, Y.; Yasuko, T.; Takeshi, O.; Norifusa, M. Fungicidal activity of the novel fungicide cyazofamid against Phytophthora infestans and other plant pathogenic fungi in vitro. Pestic. Biochem. Physiol. 2001, 71, 92-99.

13. Yang, J.C.; Wu, Q.; Liu, R.L.; Ma, S.C.; Liu, C.L. Recent advances on the development of agricultural fungicides. Agrochemicals 2008, 47, 402-405.

14. Wang, M.Y.; Li, Z.M. Synthesis and Bioactivities of Novel 2-Methoxycarbonyl-5-arylazomethine Benzenesulfonamide. Chin. J. Synth. Chem. 2012, 20, 65-68.

15. Zeng, D.Q.; Zhang, Y.M.; Chen, J. Studies on the synthesis and fungicidal activity of $N$-phenylsulfonyl- $\alpha$-triazolyl-pinacolone-hydrazone. J. Guangxi Agric. Biol. Sci. 2004, 23, 313-315.

16. Zhong, Z.M.; Chen, R.; Xing, R.G.; Chen, X.L.; Liu, S.; Guo, Z.Y.; Ji, X.; Wang, L.; Li, P.C. Synthesis and antifungal properties of sulfanilamide derivatives of chitosan. Carbohydr. Res. 2007, 342, 2390-2395.

17. Liang, X.M.; Fang, X.Q.; Dong, Y.H.; Yuan, H.Z.; Qi, S.H.; Wang, D.Q.; Wu, J.P. Synthesis and fungicidal activity of $N$-(arylsulfonylaminoethyl)-1,6-caprolactam. Chin. J. Pestic. Sci. 2008, 10, 156-160.

18. Chen, Q.W.; Shen, D.L. Synthesis and biological activity of novel 2-thiazolidiones sulfonylurea derivatives. J. Zhejiang Univ. Technol. 2008, 36, 562-564.

19. Wang, X.P.; Wang, D.Q. Synthesis and antifungal activity of $N$-substituted-2oxocyclododecylsulphonamides. Chem. J. Chin. Univ. 1997, 18, 889-893.

20. Li, X.H.; Yang, X.L.; Ling, Y.; Fan, Z.J.; Wang, D.Q.; Li, Z.M. The Synthesis and fungicidal Activity of Novel $\alpha$-Oxocycloalkylsulfonylureas. J. Agric. Food Chem. 2005, 53, 2202-2206.

21. Li, X.H.; Yang, X.L.; Liang, X.M.; Kai, Z.P.; Yuan, H.Z.; Wang, D.Q. Synthesis and biological activities of 2-oxocycloalkylsulfonamides. Bioorg. Med. Chem. 2008, 16, 4538-4544.

22. Yang, H.Y.; Yan, X.J.; Yuan, H.Z.; Liang, X.M.; Zhang, J.J.; Wang, D.Q. Synthesis and fungicidal activity of 2-oxocyclohexylsulfonamides containing fluorine. Chin. J. Pestic. Sci. 2010, 12, 449-452.

23. Li, X.H.; Ji, M.S.; Qi, Z.Q.; Li, X.W.; Shen, Y.X.; Gu, Z.M.; Zhang, Y; Wei, S.H.; Wang, Y.Z.; Wang, D.Q. Synthesis of 2-amino-6-oxocyclohexenylsulfonamides and their activity against Botrytis cinerea. Pest Manag. Sci. 2011, 67, 986-992.

24. Liang, X.M.; Wang, D.Q.; Yang, H.Y.; Wu, J.P.; Zhang, J.J.; Yan, X.J. Preparation method of 1-oxotetralyl-2-sulfonamide and its usage as a fungicide. Patent CN 101,503,381, 12 August 2009.

25. Li, X.H.; Wu, D.C.; Qi, Z.Q.; Li, X.W.; Gu, Z.M.; Wei, S.H.; Zhang, Y; Wang, Y.Z.; Ji, M.S. Synthesis, fungicidal activity, and structure-activity relationship of 2-oxo and 2-hydroxycycloalkylsulfonamides. J. Agric. Food Chem. 2010, 58, 11384-11389.

26. Li, X.H.; Wu, D.C.; Qi, Z.Q.; Gu, Z.M.; Li, X.W.; Ji, M.S. Bactericidal activity of 2-oxo and 2-hydroxycycloalkylsulfonamide to 14 kinds of pathogenic fungi. Chin. J. Pestic. Sci. 2011, 13, $423-426$. 
27. Li, X.H.; Pan, Q.; Cui, Z.N.; Ji, M.S.; Qi, Z.Q. Synthesis and Fungicidal Activity of $\mathrm{N}$-(2,4,5-trichlorophenyl)-2-Oxo- and 2-Hydroxycycloalkylsulfonamides. Lett. Drug Des. Discov. 2013, 10, 353-359.

28. Sano, T.; Ohashi, K.; Oriyama T. Remarkably fast acylation of alcohols with benzoyl chloride promoted by TMEDA. Synthesis 1999, 7, 1141-1144.

(C) 2013 by the authors; licensee MDPI, Basel, Switzerland. This article is an open access article distributed under the terms and conditions of the Creative Commons Attribution license (http://creativecommons.org/licenses/by/3.0/). 